\title{
Albinismo parcial em cutia Dasyprocta azarae (Lichtenstein, 1823) (Rodentia, Dasyproctidae), no sul do Brasil
}

\author{
Stefan Vilges de Oliveira \\ ONG ITA, Interação de Trabalhos Ambientais \\ Caixa Postal 05, CEP 96570-000, Caçapava do Sul - RS, Brasil \\ stefanbio@yahoo.com.br
}

Submetido em 02/07/2009

Aceito para publicação 08/08/2009

\section{Resumo}

O presente trabalho registra um caso de albinismo parcial em cutia, Dasyprocta azarae, observado no município de Caçapava do Sul, RS.

Unitermos: albinismo parcial, Dasyprocta azarae, sul do Brasil

\section{Abstract}

Partial albinism in agouti Dasyprocta azarae (Lichtenstein, 1823) (Rodentia, Dasyproctidae) in Southern Brazil. This work reports the partial albinism in agouti, Dasyprocta azarae, observed in city of Caçapava do Sul, RS.

Key words: Dasyprocta azarae, partial albinism, southern Brazil

O albinismo é uma anomalia genética recessiva causada pela deficiência na produção de melanina. Pode ser completa, quando ocorre a ausência total de pigmentação, ou parcial, quando a despigmentação ocorre na pele ou em parte do corpo, ou mesmo na forma de manchas (Sazima e Pombal, 1986; Sazima e Di Bernardo, 1991; Rodrigues et al., 1999; Geiger e Pacheco, 2006). Segundo Walter (1938), o albinismo é um evento raro, principalmente em populações silvestres, e somente um em cada vinte mil indivíduos apresenta alguma forma de albinismo. Indivíduos albinos, em condições naturais, normalmente são selecionados negativamente em função de sua suscetibilidade (Parsons e Bondrup-Nielsen, 1995).
Mamíferos sul-americanos com albinismo são relatados para diversos grupos, como morcegos (Ochoa e Sanchez, 1988; Moreira et al., 1992; Veiga e Oliveira, 1995; Geiger e Pacheco, 2006), roedores de pequeno porte (Pessoa e dos Reis, 1995; Cademartori e Pacheco, 1999) e médio porte, como cutias e pacas (Veiga, 1994), primatas (Veiga, 1994) e cervídeos (Veiga, 1994; Rodrigues et al., 1999).

A cutia é um roedor de médio porte da família Dasyproctidae, que vive em matas e capoeiras (Eisenberg e Redford, 1999), de ampla distribuição geográfica. Ocorre desde o centro-leste e sul do Brasil, Paraguai, até o nordeste da Argentina (Woods, 1993). No Brasil, distribui-se desde o Mato Grosso até o Rio 
Grande do Sul (Christoff, 2004). Em regiões litorâneas, há registro apenas para o Paraná (Ximenes, 1999). Possui o corpo delgado, a cabeça alongada com orelhas relativamente pequenas e membros anteriores menores que os posteriores; a massa corporal varia de 1,5 a 2,8kg; as longas extremidades posteriores tornam a cutia uma saltadora ágil. Os pêlos são ásperos, duros e longos, com coloração marrom avermelhada, dorsalmente, e região ventral mais clara (Silva, 1984).

Atualmente, os principais fatores que levam as populações de cutias ao declínio no sul do Brasil, são a destruição e descaracterização de habitats, bem como a caça predatória (Christoff, 2004).

O presente registro vem contribuir com informações acerca da ocorrência de albinismo parcial em Dasyprocta azarae no sul do Brasil.

\section{Descrição da ocorrência}

Um exemplar de cutia, Dasyprocta azarae (Figura 1), foi encontrado no Bioma Pampa, região geomorfológica da Serra do Sudeste, no município de Caçapava do Sul, RS, na localidade denominada de Serra da IMEC, Caieiras (30³1'31,0"S; $\left.53^{\circ} 26^{\prime} 55,4^{\prime \prime} \mathrm{W}\right)$, em uma propriedade agrícola, próxima ao cultivo de milho. O local apresenta relevo topograficamente acidentado com declividade superior a $60^{\circ}$, e na base desta elevação há um pequeno riacho, situado a aproximadamente $300 \mathrm{~m}$ do local onde ocorreu a captura. $\mathrm{O}$ indivíduo foi capturado em janeiro de 2008, por um morador da zona rural, com uma armadilha tomahawk, de 70x70x40 cm, utilizando como isca grãos de milho. O espécime albino parcial, uma fêmea adulta, apresentava as mamas ingurgitadas, pelagem branca, pele, pés garras e rostro rosados e olhos castanhos claros, caracterizando-a como albino parcial por não apresentar olhos vermelhos.

$\mathrm{O}$ agricultor relatou que no mesmo período coletou mais sete indivíduos com pelagem padrão e que utiliza esses animais como alimento, caçando-os. Segundo o agricultor "o indivíduo albino parcial era avistado com frequência e, aparentemente, era mais tolerante a aproximação de pessoas e animais domésticos do que os indivíduos de pelagem padrão".

A visualização e captura das cutias é frequente nos meses mais quentes, período no qual facilmente se encontram os indivíduos aos pares. Na época em que foi capturada, a cutia apresentava alopecia e manchas vermelhas pelo corpo, sendo provavelmente algum tipo de micose ou dermatite (Figura 2).

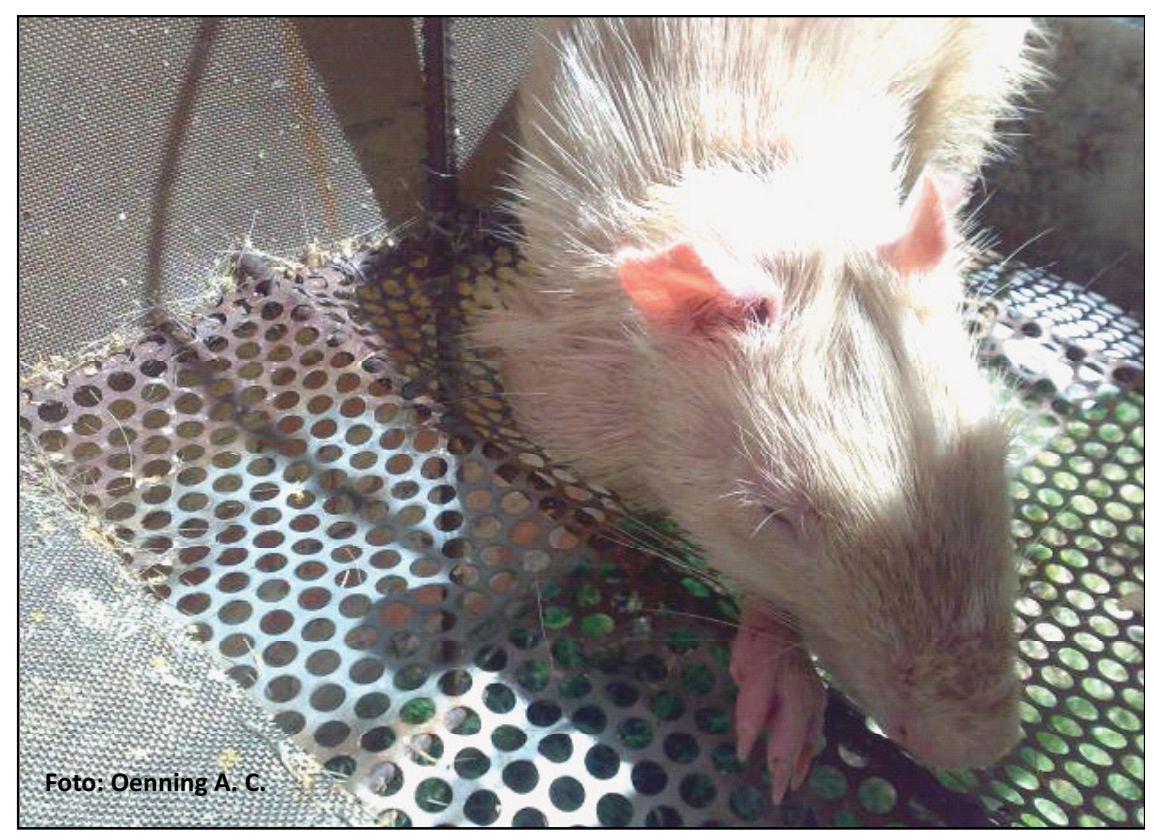

FIGURA 1: Dasyprocta azarae com albinismo parcial (Cativeiro). 


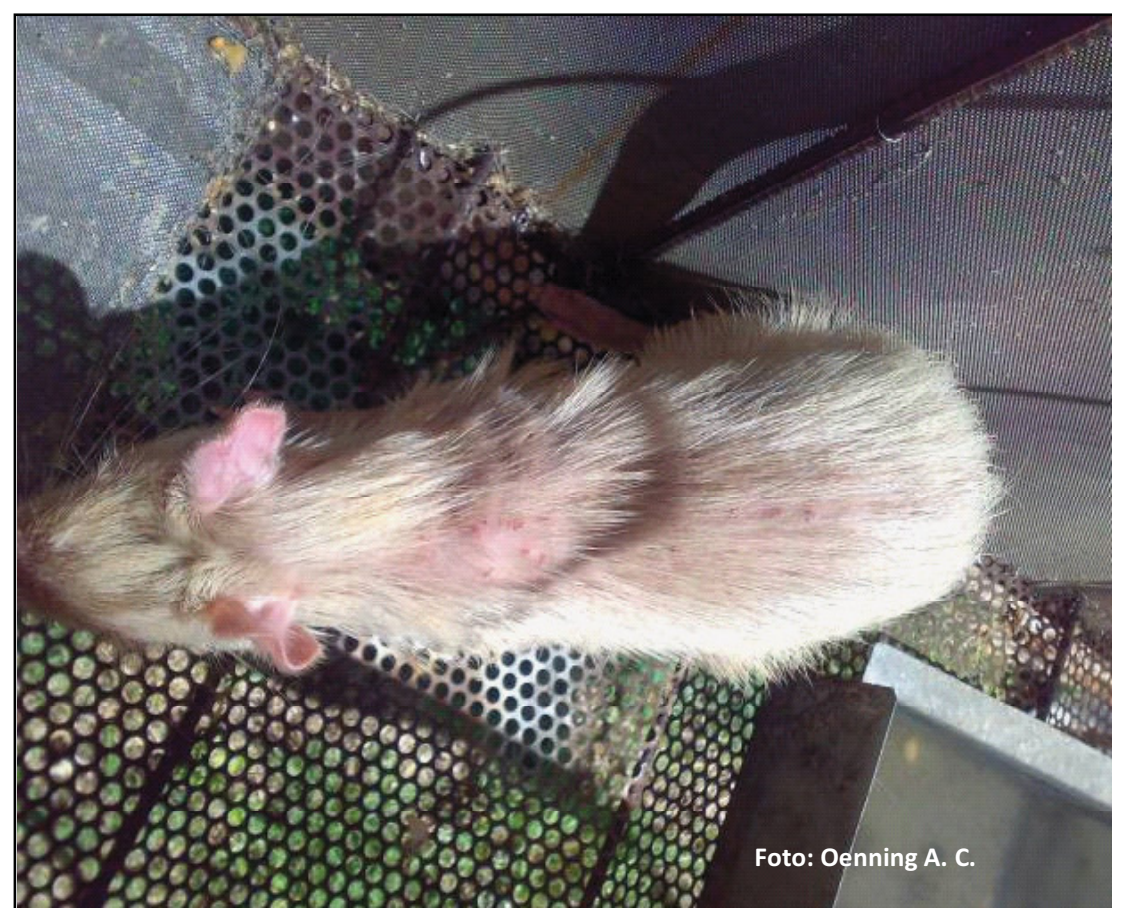

FIGURA 2: Dasyprocta azarae com alopecia na região dorsal.

A fêmea permaneceu em cativeiro por aproximadamente 60 dias. $\mathrm{O}$ animal era alimentado com grãos, vegetais e frutas, e mantido isolado, pois quando em contato com outros de pelagem padrão havia agressão, sendo que as cutias normais arrancavam os pêlos da região dorsal, agravando mais a alopecia. Neste período, o animal recuperou-se até que foi abatido para o consumo.

\section{Agradecimentos}

Em especial, agradeço a Susi M. Pacheco, Lize H. Cappellari pelas valiosas correções dadas ao manuscrito; ao Alexandre (Chacal), ao Sr. Ademar e à Sra. Tereza pelas informações referentes ao episódio; à Ana Claudia Oenning pelo registro fotográfico; e aos pesquisadores que anteriormente relataram episódios de albinismo, sem o qual o manuscrito não possuiria o mesmo valor.

\section{Referências}

Brismar, J.; Harfi, H. A. 1992. Partial albinism with immunodeficiency: A rare syndrome with prominent posterior fossa white matter changes. American Journal of Neuroradiology, 13 (1): 387-393.
Cademartori, C. V.; Pacheco, S. M. 1999. Registro de albinismo em Delomys dorsalis (Hensel, 1872) (Cricetidae, Sigmodontinae). Biociências, 7 (1): 195-197.

Christoff, A. U. 2004. Roedores e Lagomorfos. In: Fontana, C. S.; Bencke, G. A. \& Reis, R. E. Livro vermelho da fauna ameaçada de extinção no Rio Grande do Sul. EDIPUCRS, Porto Alegre, Brasil, p.567-571.

Eisenberg, J. F.; Redford, K. H. 1999. Mammals of the neotropics, the central neotropics: Ecuador, Peru, Bolivia e Brasil. v.3. University of Chicago Press, Chicago, USA, 609pp.

Geiger, D.; Pacheco, S. M. 2006. Registro de albinismo parcial em Nyctinomops laticaudatus (e. Geoffroy, 1805) (Chiroptera: Molossidae) no Sul do Brasil. Chiroptera Neotropical, 12 (1): 250-254.

Jeffery, G.; Williams, A. 1994. Is abnormal retinal development in albinism only a mammalian problem? Normality of a hypopigmented avian retina. Experimental Brain Research, 100 (1): 47-57.

Klein, N. P.; Le-Deist, F. S.; Fraitag, S.; Prost, C.; Durandy, A.; Fischer, A. ; Gricelli, C. 1994. 1994. Partial albinism with immunodeficiency (Gricelli syndrome). Journal Pediatrics, 125 (6): 886-895.

Moreira, E. C.; Silva, M. C. P.; Veloso, J. G. 1992. Albinismo em Desmodus rotundus rotundus, Quiróptera (E. Geofroy, 1810). Arquivo Brasileiro de Medicina Veterinária e Zootecnia, 44: 549-552. 
Ochoa, J.; Sanchez, H. J. 1988. Lonchorina fernandezi, new record (Chiroptera Phyllostomidae) for venezuela, with some obeservations on its biology. Memorias de la Sociedad de Ciencias Naturales La Salle, 48 (129): 133-154.

Parsons, G. J. ; Bondrup-Nielsen, S. 1995. Partial albinism in an island population of Meadow Voles, Microtus pennsylvanicus, from Nova Scotia. The Canadian Field-Naturalist, 109 (2): 263-264.

Pessoa, A. L.; dos Reis, S. F. 1995. Coat color variation in Proechimys albispinus (Geoffroy, 1838) (Rodentia, Echimyidae). Boletim do Museu Nacional, Nova Série Zoologia, 361: 1-5.

Rodrigues, F. H. G.; Silveira, L.; Jácomo, T. A.; Monteiro-Filho, E. L. A. 1999. Um albino parcial de veado campeiro (Ozotoceros bezoarticus, Linnaeus) no Parque nacional das Emas, Goiás. Revista Brasileira de Zoologia, 16 (4): 1229-1232.

Sanyal, S.; Zeilmaker, G. H. 1988. Retinal damage by constant light in chimeric mice: Implications for the protective role of melanin. Experimental Eye Research, 46 (5): 731-744.

Sazima, I.; Pombal J. Jr. 1986. Um albino de Rhamdella minuta, com notas sobre comportamento (Osteichthyes, Pimelodidae). Revista Brasileira de Biologia, 46 (2): 377-381.
Sazima, I.; Di Bernardo, M. 1991. Albinismo em serpentes neotropicais. Memórias do Instituto Butantan, 53 (2): 167-173.

Silva, F. 1984. Mamíferos silvestres do Rio Grande do Sul. Fundação Zoobotânica do Rio Grande do Sul, Porto Alegre, Brasil, 246pp.

Veiga, L. A. 1994. Um caso de albinismo em Tayassu tajacu Linnaeus (Artiodactyla, Tayassuidae) na Serra do Mar São José dos Pinhais, Paraná. Revista Brasilileira de Zoologia, 11 (2): 341 343.

Veiga, L. A.; Oliveira, A. T. D. 1995. A case of true albinism in the bat Molossus molossus, Pallas (Chiroptera, Molossidae) in Santa Vitória do Palmar, RS, Brazil. Arquivos de Biologia e Tecnolologia, 38 (3): 879-881.

Walter, H. E. 1938. Genetics: And introduction of study of heredity. Mac-Millan, New York, USA, 422pp.

Woods, C. A. 1993. Hystricognathi. In: Wilson, D. E. \& Reeder, D. A. (Eds). Mammals species of the world. A taxonomic and geographic reference. $2^{\text {nd }}$ ed. Smithsonian Institution Press, American Society of Mammalogist, Washington, USA, p.771-806

Ximenes, G. E. I. 1999. Sistemática da Família Dasyproctídae Bonaparte, 1938(Rodentia, Histricognathi) no Brasil. Dissertação de Mestrado, Universidade de São Paulo, Brasil, 429pp. 